\title{
Desarrollo de una herramienta didáctica basada en realidad extendida para un curso de física en ingeniería ambiental
}

\section{Development of a teaching tool based on extended reality for a physics course in environmental engineering}

\author{
Alex Fabián Cadena Cairasco ${ }^{+(D)}$, Mario Alberto Jurado Eraso*(i) y \\ Pablo José Pabón Santacruz* ${ }^{+}$ \\ +Ingeniería Ambiental, Universidad Mariana, Pasto, Colombia \\ *Grupo de Investigación Ambiental GIA, Universidad Mariana, \\ Pasto, Colombia
}

Resumen. Debido a las dificultades identificadas por varios años en los procesos de enseñanzaaprendizaje y evaluación del concepto de energía en el programa de Ingeniería Ambiental de la Universidad Mariana en Pasto (Nariño-Colombia), principalmente por tratarse de un concepto abstracto y de que su comprobación no puede determinarse de manera directa; en esta investigación se exploró algunas potencialidades de la realidad extendida en dicho proceso, mediante el desarrollo de una herramienta didáctica, para el curso de física, que consistió en la programación de un entorno virtual, que simula un recorrido en una montaña rusa, seguido de una clase en un entorno de realidad aumentada, guiada a través de un proceso de interacción con objetos en movimiento y textos interactivos. La herramienta desarrollada tiene el potencial de mejorar la experiencia del estudiante estimulando sus sensaciones, sin poner en riesgo su integridad y sin la necesidad de inversión en infraestructura.

Se presentan en este trabajo los resultados obtenidos en las primeras tapas de una investigación proyectiva y la evaluación parcial de la misma, mostrando la potencialidad de la realidad aumentada y virtual dentro de los procesos de enseñanza y aprendizaje, como una herramienta de nueva generación que permite una mejor retención de la información y adaptación a los canales de comunicación de los estudiantes.

Palabras Claves. Realidad aumentada; realidad virtual; Educación; Aprendizaje; Física; Energía.

\begin{abstract}
Due to the difficulties identified for several years in the teaching-learning processes and evaluation of the concept of energy in the Environmental Engineering program of the Mariana University in Pasto (Nariño-Colombia), mainly because it is an abstract concept and that it is Verification cannot be determined directly; In this research it was explored some potentialities of the extended reality, through the development of a didactic tool for the physics course, which consisted of programming a virtual environment, that makes a simulates a journey on a roller coaster, followed by a class in an augmented reality environment, guided through a process of interaction with moving objects and interactive texts. The developed tool has the
\end{abstract}


potential to improve the student experience by stimulating their feelings, without compromising their integrity and without the need for investment in infrastructure.

In this document presents the results obtained in the first stage of a projective investigation and a partial evaluation of this, also showing the potential of augmented and virtual reality within the teaching and learning processes, as a new generation tool that allows better retention of information and adaptation to student communication.

Keywords. Augmented reality; virtual reality; education; learning; physics; energy.

Como citar. A. F. Cadena Cairasco, M. A. Jurado Eraso y P. J.e Pabón Santacruz, "Desarrollo de una herramienta didáctica basada en realidad extendida para un curso de física en ingeniería ambiental", Jou. Cie. Ing, vol. 12, no 1, pp. 202-214, 2020. doi:10.46571/JCI.2020.1.18

Recibido: 13/03/2020 Revisado: 23/06/2020 Aceptado: 07/07/2020

\section{Introducción}

Una aplicación móvil o app, es un software diseñado para funcionar en teléfonos inteligentes y otros dispositivos móviles. Hay que mencionar además que, hoy en día cada vez es más fácil para cualquier persona acceder a los dispositivos móviles y celulares inteligentes, pues existen diferentes facilidades de pago y servicios de conexión. Así mismo, se sabe que existen muchos aplicativos que le permiten a los usuarios el desarrollo de diversas actividades como el pago de servicios, consultas médicas o educación [1].

Es así como el uso de estas herramientas supone un proceso de democratización del acceso a la información y al conocimiento y les permiten a las personas la solución de todo tipo de problemas de forma casi inmediata [1], [2]. Así pues, en el contexto académico y en específico en relación con los procesos de enseñanza-aprendizaje que tienen que ver con los cursos del área de la física; esto es, física, termodinámica o fisicoquímica, en las que tradicionalmente los estudiantes encuentran muchas dificultades por tratarse de cursos que tienen muchos conceptos abstractos y para los cuales los estudiantes normalmente no han tenido una formación previa adecuada, este tipo de herramientas se convierten en una alternativa bastante atractiva para superar tales limitaciones.

En este contexto, considerando especialmente el interés de los jóvenes de hoy en día por aquellas aplicaciones que tienen que ver con juegos realistas; la herramienta de realidad virtual, permite acceder de manera amigable a los estudiantes y les otorgará una apropiación de las temáticas, especialmente que tienen que ver con la energía y sus transformaciones, a través de la experimentación de sensaciones y la medición de variables en un entorno virtual.

También es de destacar que esta propuesta resulta conveniente en el fortalecimiento y enriquecimiento de los procesos de enseñanza-aprendizaje significativos propuestos desde una perspectiva de los programas de Ingeniería en relación con la calidad requerida por los entes normalizadores competentes a nivel estatal y en concordancia con su misión y visión de las instituciones educativas.

Así mismo, se relaciona con las necesidades actuales en torno a la minimización del uso de recursos físicos, económicos y energía a nivel local y global, pues con el desarrollo de prácticas no convencionales alrededor de la realidad virtual y aumentada se evita la construcción de nuevas áreas para laboratorio, el uso de equipos, materiales y reactivos, que necesariamente demandan recursos y energía y que muchas veces pueden poner en riesgo la integridad física de los estudiantes; así mismo, permite beneficiar a mucha de la población diversa que hace parte de una institución educativa; esto es, por ejemplo: estudiantes con alguna limitación física [3] [6].

Por otra parte, vale la pena mencionar que, para los estudiantes de ingeniería, temas como la energía y sus transformaciones resultan bastante difíciles de aprehender pues son abstractos 
y su medición no se puede determinar de manera directa sino a través de otras variables como la posición de un cuerpo o su temperatura, y por lo tanto, los estudiantes tienden a confundirse, resultando en un proceso inapropiado del aprendizaje de los fenómenos asociados y que resultan fundamentales para el estudio de áreas como: los balances de masa y energía, la mecánica de fluidos, la hidrología o la modelación ambiental, entre otros asuntos importantes para el quehacer de un ingeniero.

En resumen, los resultados derivados de esta investigación sobran en beneficio tanto de estudiantes como de los docentes y de igual forma pueden ser un aporte para fortalecer los procesos de enseñanza-aprendizaje a nivel institucional. Al respecto, experiencias con el uso de realidad virtual han resultado exitosas a nivel mundial y todavía se siguen desarrollando herramientas en este sentido, por ejemplo, en áreas como la formación en medicina e ingeniería. En este sentido, los estudiantes, experimentan con sensaciones, equipos y acciones que son difíciles de realizar en vivo y que se pueden repetir una y otra vez, terminando por asimilar por analogía las teorías alrededor de asuntos muy difíciles o abstractos [6].

En el aprendizaje de la física un estudiante se enfrenta a la exploración y entendimiento de cómo funciona el mundo a su alrededor, con lo que se ve involucrado en el estudio de teorías que de manera matemática describen cómo funciona un sistema físico. A parte de la descripción y predicción de un fenómeno en particular está la apropiación propia del concepto que define al fenómeno, con lo que dentro de la didáctica se busca explorar lo que sabe un estudiante y a partir de ello reorientarlo a una aproximación más científica del mismo [7].

De este modo, dentro del campo didáctico de las ciencias esta la exploración de las diferentes formas que tienen las matemáticas para poderse representar, lo cual se aplica al campo de la física ya que hace uso de ellas como herramienta fundamental, por tanto el estudio de las representaciones semióticas, donde estas "Hacen referencia a todas aquellas construcciones de sistemas de expresión y representación que pueden incluir diferentes sistemas de escritura, como números, notaciones simbólicas, representaciones tridimensionales, gráficas, redes, diagramas, esquemas, etc. y cumplen funciones de comunicación, expresión, objetivación y tratamiento" [8], se ven involucradas en esta investigación, ya que será a partir de algunas de ellas que se acercara al estudiante a la apropiación de un concepto especifico como es la energía.

Con lo anterior, dado que se busca darle solución a una necesidad práctica centrada en los procesos de enseñanza-aprendizaje dentro del área de física en las carreras de ingeniera, esta investigación es de tipo proyectiva, con lo que en este sentido se enmarca la exploración de la necesidad, el diseño y desarrollo de la mejor solución, y la evaluación de los resultados.

En consideración a estos apartados, en la propuesta de realidad virtual y aumentada se sitúa al alumno en un entorno donde se combinan el mundo real y el mundo digital, en el que encuentra algunas situaciones relacionadas con el concepto de energía, como es un recorrido histórico de la evolución del concepto, la indagación de ideas previas, un acercamiento más profundo sobre lo que es la energía mecánica y una evaluación del posible cambio conceptual.

Una vez teniendo clara la importancia de introducir este tipo de tecnologías en la educación, el proyecto de investigación enfocado en crear una didáctica utilizando este tipo de herramientas, se vislumbra como una herramienta didáctica para el aprendizaje de las ciencias físicas, mediante la cual se realiza un manejo incluyente de los conceptos digitales para un mejor aprendizaje, dentro de la implementación se desarrolla ambientes virtuales de visualización concerniente a fenómenos físicos.

Los ambientes de realidad aumentada desarrollados para la investigación, contienen visualizaciones de fenómenos como la gravedad, inercia, energía potencial y energía cinética; además podemos citar otros componentes de gran relevancia dentro de la aplicación, los cuales son los botones interactivos con el usuario, estos permiten la manipulación de objetos virtuales llevando a la compresión de los fenómenos físicos en diversas experimentaciones, creando en el estudiante una nueva perspectiva y un gran interés por el tema a tratar. 
Dentro de la aplicación referente a realidad virtual el entorno de inmersión proporciona al estudiante una realidad de infinitas posibilidades, sensaciones y experiencias, para el caso en particular del proyecto de investigación el entorno consiste en una montaña rusa en la cual se describe los diversos valores que adoptan las magnitudes de energía y velocidad, el efecto de experimentar estas magnitudes en un entorno de inmersión estimula la captación del conocimiento a través de los sentidos involucrados en la experimentación.

\section{Metodología}

La metodología que se usó está ligada a tres etapas dentro de la investigación proyectiva, la cual consiste en encontrar la solución a los problemas prácticos, se ocupa de cómo deberían ser las cosas para alcanzar los fines y funcionar adecuadamente [28]. Siendo la educación y todos los procesos involucrados en la misma un tema de gran relevancia, esta investigación plantea y pone en marcha la adaptación a la realidad virtual y aumentada conceptos poco visibles y nada tangibles que muchas veces son netamente teóricos y aburridos para los estudiantes; esta adaptación permitirá incrementar el rendimiento escolar así como lo ha demostrado la Unidad LabHuman, del Instituto I3BH, en la Universidad Politécnica de Valencia [29].

Esta investigación fue encaminada a la proyección dado que está fundamentada en la elaboración de una propuesta o de un modelo, para solucionar problemas o necesidades de tipo práctico, ya sea de un grupo social, institución, un área en particular del conocimiento, partiendo de un diagnóstico preciso de las necesidades del momento, los procesos explicativos o generadores involucrados y las tendencias futuras [28]. Las faces para este tipo de investigación son varias, pero se adaptaron a tres de las siguientes etapas:

\section{Etapa 1. Exploración de la necesidad}

Esta etapa está enmarcada a la exploración de las dificultades que se presenta en la apropiación conceptual de la energía de los estudiantes en los primeros semestres de las carreras de ingeniería dentro de la Universidad Mariana Pasto-Colombia, así como la exploración del uso de las TIC, la realidad virtual (RV) y aumentada (RA) dentro de diferentes campos académicos y no académicos.

\section{Etapa 2. Diseño y desarrollo de la mejor solución}

Identificada la necesidad y la posible adaptación de la RV y RA, en esta etapa se realiza el diseño, desarrollo y prueba de la herramienta didáctica basada en RV y RA.

\section{Etapa 3. Evaluación de los resultados parciales en una prueba piloto}

Se determina la potencialidad de la unidad didáctica basada en RV y RA a partir de las opiniones que realizaron algunos participantes del proyecto durante las pruebas piloto con estudiantes y docentes. Para este trabajo se presenta de manera parcial la evaluación de los criterios encaminados a la inmersión, la satisfacción, aprendizaje, efectividad, motivación, emoción y socialización.

\section{Resultados}

\subsection{Etapa 1 - Exploración de la necesidad}

3.1.1. Dificultades en el proceso de enseñanza-aprendizaje del concepto de energía Para muchos no debe ser desconocido que al momento en el que se pasa de una formación básica a una profesional, las teorías, conceptos y demás términos vistos en la universidad tienden a ser más específicos y por tanto más complejos. Es en este sentido, en el que los estudiantes de ingeniería en sus primeros años de estudio se ven inmersos en el aprendizaje de ciencias como pilares fundamentales de su carrera y la física no se escapa de sus estudios.

En el aprendizaje de la física todo aquel que haya tenido el gusto de explorar este vasto mundo de conocimientos, se habrá enfrentado a de diversas teorías y leyes que describen, modelan y predicen el comportamiento de algún fenómeno del universo observable. Es aquí donde el estudiante muchas veces se encuentra con conceptos que describen fenómenos que no 
son tangibles y por lo general son medibles pero no siempre de manera directa. Al hablar de energía se encuentra que esta presenta dificultades motivadas desde la perspectiva y el sentido común. Pacca y Herrique [9], articulando varios estudios sobre las concepciones en el estudio de la energía muestran que los estudiantes traen consigo ideas previas relacionadas con: La energía como causa o fuente; la energía como resultado de un movimiento o acción y la energía como sustancia o ingrediente.

Así mismo estos autores muestran que una vez impartidos los contenidos fundamentales del concepto se presentan dificultades como: "a) interpretar los fenómenos en términos de propiedades absolutas o cualidades intrínsecas a un objeto en detrimento de posibles interacciones entre elementos de un sistema; b) buscar o atribuir causas a cualquier acción o movimiento observado en un fenómeno cualquiera; c) materializar o substancializar entidades abstractas, que son poco comprendidas" [9, p. 160].

Es de destacar que desde el descubrimiento de este concepto y su fundamentación, solo tiene significado hablar de este en el sentido de su conservación o transformación, donde se ven involucrados principalmente, la observación de interacciones físicas, la delimitación de sistemas de cuerpos o sistemas físicos y la caracterización de los estados de esos sistemas [10].

En este orden de ideas este proyecto se enmarca en los primeros acercamientos que se tiene el estudiante al interactuar con el concepto, donde se puede encontrar con la conservación de la energía mecánica en la interacción que tienen los objetos con la tierra, donde explícitamente se ven involucradas las transformaciones entre de energía potencial gravitacional, energía cinética y manifestaciones de otras energías (Calor). Como se mencionó anteriormente los estudiantes tienden a relacionar cantidades de energía, como es el asociar que si un objeto está a cierta altura este tiene una cantidad de energía potencial o algo similar cuando este está en movimiento asociándole una cantidad de energía cinética, esto muchas veces se ve complementado con la interacción que se tiene con la fundamentación matemática de este concepto y los estudiantes solo realizan remplazos sobre las mismas para comprobar esas cantidades.

Tomando algunos trabajos donde se enfatiza el desarrollo de unidades y actividades para el concepto de energía, trabajo y conservación, todas convergen en que se debe iniciar con estudios epistemológicos e históricos de este concepto mostrándolo como un eje unificador de toda la física y otras ciencias, así también concuerdan que el desarrollo de las actividades que propone el docente, deben instruir a partir de las concepciones que traen los chicos en sus saberes previos, dando a entender que muchas de estas por ser espontaneas, no formales o traídas de la cotidianidad, producen malinterpretaciones en el transcurso del curso o en otros, con lo que sugieren tener en cuenta los objetivos y metas de la apropiación de este concepto que se ven manifestados en los proyectos educativos nacionales o institucionales [11]- [14].

Con lo mencionado, es de interpretar que existen un número considerable de dificultades en el aprendizaje del concepto de energía, manifestado desde el concepto mismo, el uso verbal adecuado cuando se habla de él sobre un sistema determinado y todas aquellas representaciones que se pueden manifestar al resolver un problema o al aplicarlo dentro de un laboratorio, así con esto permitió enfocarse en la interpretación grafica de la transformaciones de energía que se presenta en sistemas mecánicos aislados. Como lo mencionan varios autores todas las representaciones semióticas que pueda manifestar un concepto son muy importantes en el desarrollo conceptual para un estudiante y cuando se presenta un dominio conjunto de todas estas (comunicación, expresión, objetivación y tratamiento), se puede considerar que el estudiante entiende y ase uso del concepto [8], [15]- [17].

3.1.2. Exploración del uso de las TIC y la realidad virtual $(R V)$ y aumentada (RA) En relación a la problemática expuesta, en la revisión bibliográfica se encontró que uno de los recursos favorables con los que se cuenta hoy en día es el uso de las TIC, la realidad virtual y aumentada y dentro de estos procesos se ve como una oportunidad significativa para mitigar las dificultades 
en el aprendizaje del concepto de energía. Estas herramientas modernas permiten el uso de recursos tecnológicos, como los usados en los videojuegos, estas permiten acercar a los estudiantes a teorías difíciles de aprehender a través de una realidad simulada, donde es posible por ejemplo, repetir una y otra vez la simulación, sin recurrir a gastos adicionales en asesorías o trasportes innecesarios a instalaciones como son los laboratorio [18]- [20].

Desde una perspectiva más general se ha encontrado que los usos más significativos que se le ha dado a la RA están asociados a los educativos demostrando que se dinamiza el aprendizaje y favorece la aprehensión de conocimientos de una manera divertida y lúdica siendo un elemento motivador para los chicos. También es de destacar que se encuentran usos en la medicina, navegación móvil, el marketing, los videojuegos, etc. [21]- [24].

\subsection{Etapa 2. Diseño y desarrollo de la mejor solución}

3.2.1. Diseño, desarrollo y prueba de la herramienta didáctica basada en $R V$ y $R A$ Dentro de los procesos encaminados para la ejecución de esta etapa, se determinó adquirir dispositivos como: un dispositivo móvil alta gama Samsung S8 plus, debido a sus excelentes características en cuanto al procesamiento de operaciones lógico matemáticas y excelente resolución de la pantalla, unas gafas con visión de realidad virtual Daydream View de Google (Figura 1), por su diseño liviano y comodidad para el usuario, estas incluyen un control remoto tipo puntero con el cual se puede desplazar en el entorno virtual, controlando acciones y eventos, estas gafas y el dispositivo móvil conforman la RV, y finalmente se contó con las gafas MOVERIO BT-300 de EPSON dispositivo para RA (Figura 2), este tipo de gafas cuentan con la tecnología más avanzada de Epson de pantalla digital OLED (diodo orgánico emisor de luz) con base de silicio, este tipo de tecnología permite observar dentro del lente de las gafas, una proyección de una pantalla con una gran nitidez, en esta se puede observar videos provenientes de la cámara interna el dispositivo, así como la proyección de un sistema móvil con lenguaje Android, así mismo el sistema está controlado por dispositivo tipo Android 5.1, con un procesador Intel Atom X5 de cuatro núcleos, en el cual se pueden ejecutar aplicaciones móviles, conexión a internet mediante red wifi, este dispositivo también puede utilizarse como interfaz de control para el dispositivo.
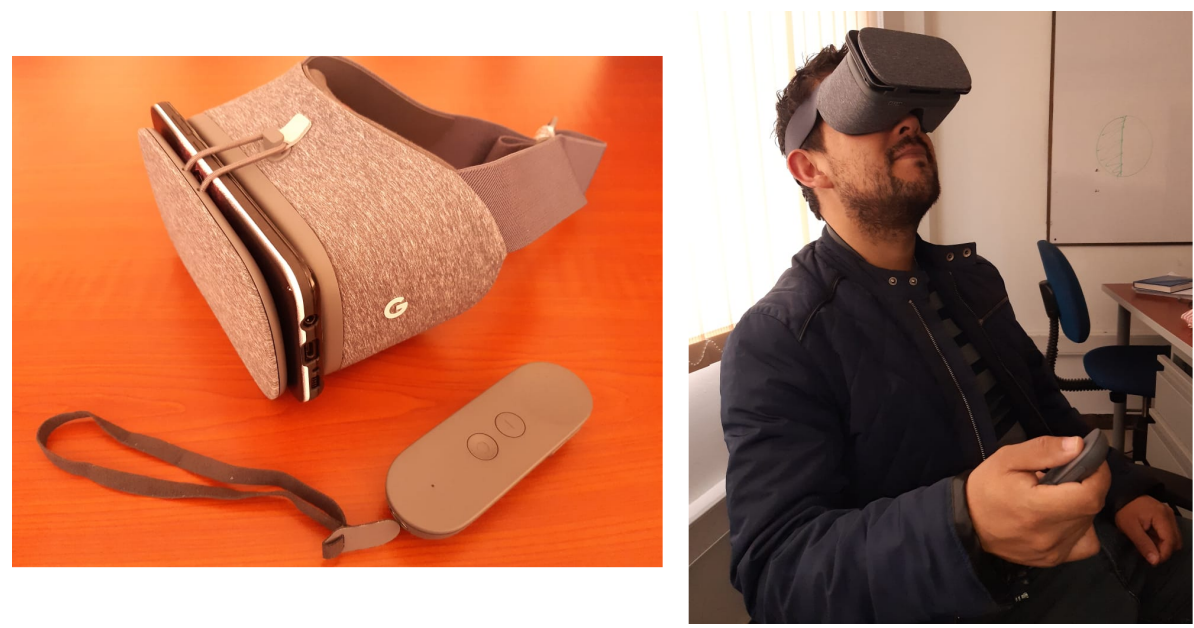

Figura 1: A la izquierda, gafas con visión de realidad virtual Daydream View de Google; A la derecha, docente en prueba de la tecnología RV.

3.2.2. Diseño estructural de la unidad didáctica para el concepto de energía. Para la elaboración de la herramienta basada en RV y RA se partió de la intención de que es lo que espera 

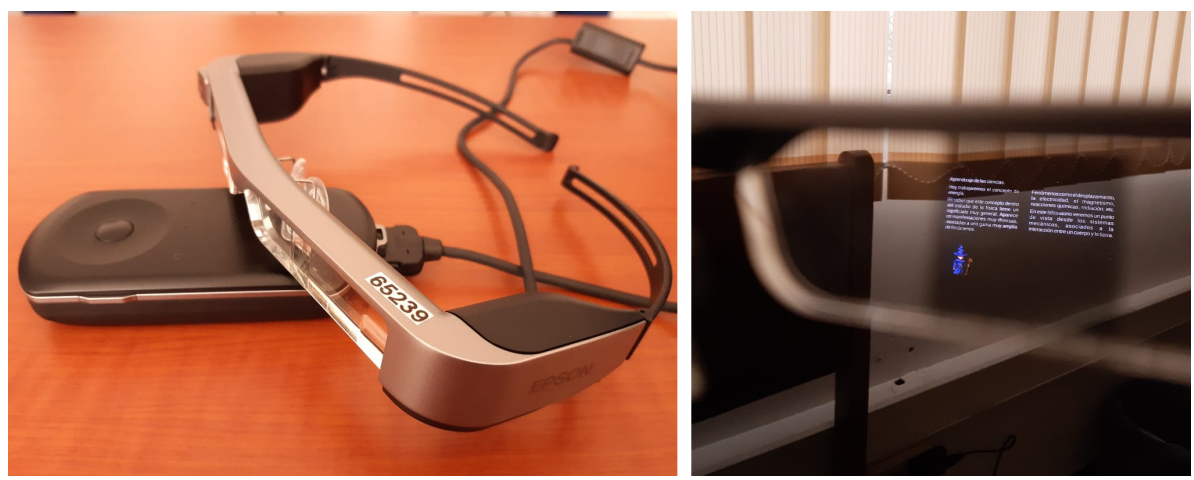

Figura 2: A la izquierda, gafas MOVERIO BT-300 de EPSON; A la derecha, vista interna de las gafas RA tipo holograma.

que los estudiantes aprendan al momento de hacer uso de ellas, por tanto se planteó como objetivo general de la unidad desarrollar una apropiación conceptual y manejo adecuado de las representaciones gráficas que posee el estudio del concepto de energía mecánica.

Como se había mencionado anteriormente debe entenderse que el estudio de las ciencias naturales y dentro del campo científico existe un variado modo de representar un concepto, desde un formalismo verbal el cual encamina a explicar de qué trata, hasta su representación como modelo matemático, a todos estos modos se le conoce como representaciones semióticas. En este sentido, nuestro equipo de trabajo toma la decisión de fomentar la transición de las representaciones del concepto de energía mecánica al de sus representaciones gráficas, siendo este de gran importancia debido a que los futuros profesionales dentro del campo de la ingeniería se enfrentan a la lectura continua de este tipo de información.

Visto de esta forma, tomado la metodología para el diseño de unidades didácticas que presentan los autores Tamayo, Orrego y Ruiz [25] y a partir de las observaciones que se realizaron sobre la investigación de Perrotta et al [13], en el que muestran paso a paso la consolidación de una estrategia didáctica sobre el concepto de energía, se estructura la secuencia didáctica que se implementara dentro de los dispositivos de RV y RA. A continuación se presenta esta estructura en el cual de manera general se evidencia la intención y el proceso de la unidad (Tabla 1).

3.2.3. Adaptación del concepto dentro de la $R V$ y RA. En este orden de ideas, esta propuesta pretende hacer uso del aprendizaje basado en RV y RA, donde Tom Caudell, uno de los pioneros en el desarrollo de propuestas de realidad aumentada encaminadas a la educación, muestra que esta metodología obliga al docente a definir todo los detalles que permitirán dar cumplimento al objetivo de la unidad que será impartida dentro de un entorno completamente digital [26], así mismo, debe permitir que el estudiante desarrolle las competencias específicas que se espera alcance al apropiarse del concepto de energía.

Definida la estructura de la unidad, se procedió a realizar el contenido se verá dentro de cada entorno virtual. También es necesario enfatizar que, nuestro proyecto hace uso del SDK (Kit de Desarrollo de Software) de realidad aumentada Vuforia, "Este SDK tiene una comunidad de más de 200.000 desarrolladores registrados en todo el mundo en su plataforma que facilita su soporte y evolución; cuenta con compatibilidad para plataformas móviles como iOS y Android, además de su fácil manejo dentro del editor de Unity3D, se pueden utilizar como marcadores, no solo códigos QR, sino también imágenes, marcadores con forma cilíndrica, forma de caja y objetos" Serrano, 2012, citado en [21, p. 107].

- Concepto de energía en el entorno de la RV 
Tabla 1: Unidad didáctica para el concepto de energía mecánica adaptado al aprendizaje basado en RV y RA.

\begin{tabular}{|l|l|}
\hline Objetivo & $\begin{array}{l}\text { Desarrollar una apropiación concep- } \\
\text { tual y manejo adecuado de las re- } \\
\text { presentaciones gráficas que posee } \\
\text { el estudio del concepto de energía } \\
\text { mecánica. }\end{array}$ \\
\hline Contenidos & $\begin{array}{l}\text { Concepto de energía como producto } \\
\text { de la transformaciones de un siste- } \\
\text { ma; } \\
\text { Energía mecánica como la variación } \\
\text { y transformación de energía poten- } \\
\text { cial, cinética y la manifestación de } \\
\text { otras } \\
\text { Gráficos de transformación de } \\
\text { energía mecánica. }\end{array}$ \\
\hline Aprendizaje basado en RV y RA. \\
\hline Metodología & $\begin{array}{l}\text { Dispositivos de RV y RA } \\
\text { Computador } \\
\text { Celular } \\
\text { Libro guía } \\
\text { Textos continuos o discontinuos }\end{array}$ \\
\hline
\end{tabular}

Se toma la iniciativa que dentro de la RV se procedería a la creación de un entorno virtual semejante al de una montaña rusa para simular su funcionamiento y poder realizar los cálculos de algunas variables de la física enfatizando las transformaciones de la energía mecánica (energía cinética y potencial), que experimenta el carrito en su recorrido al interactuar con la tierra en los trayectos de subida y bajada (Figura 3 ).

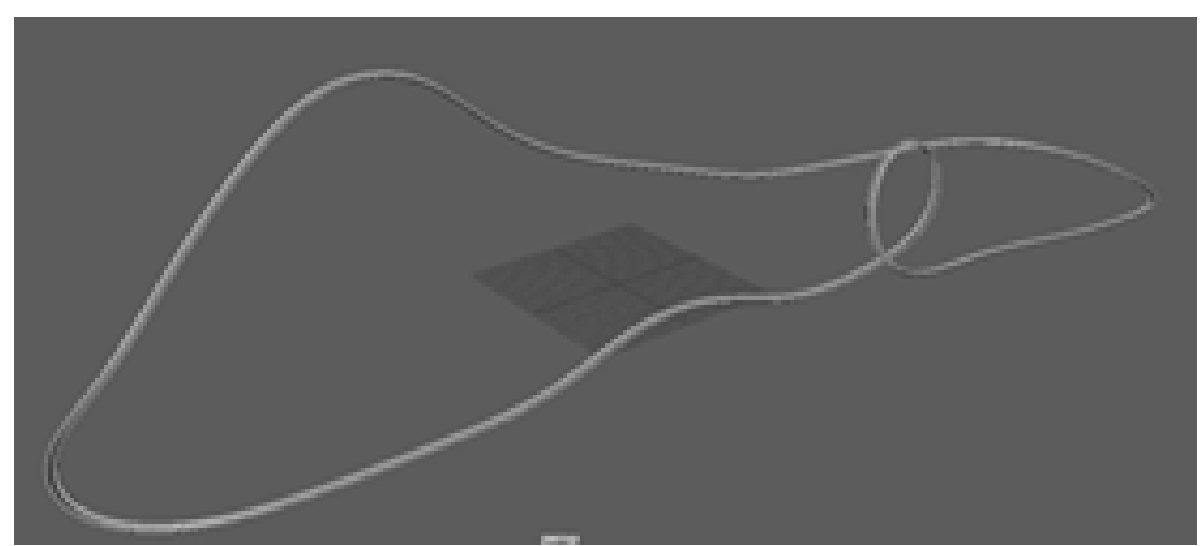

Figura 3: Vista panorámica del recorrido en montaña rusa bajo RV sin textura.

En el recorrido de la montaña rusa el usuario es pasajero sobre el carrito que se mueve sobre ella, puede manipular el moviendo de las gafas con el movimiento de su cabeza a fin de poder 


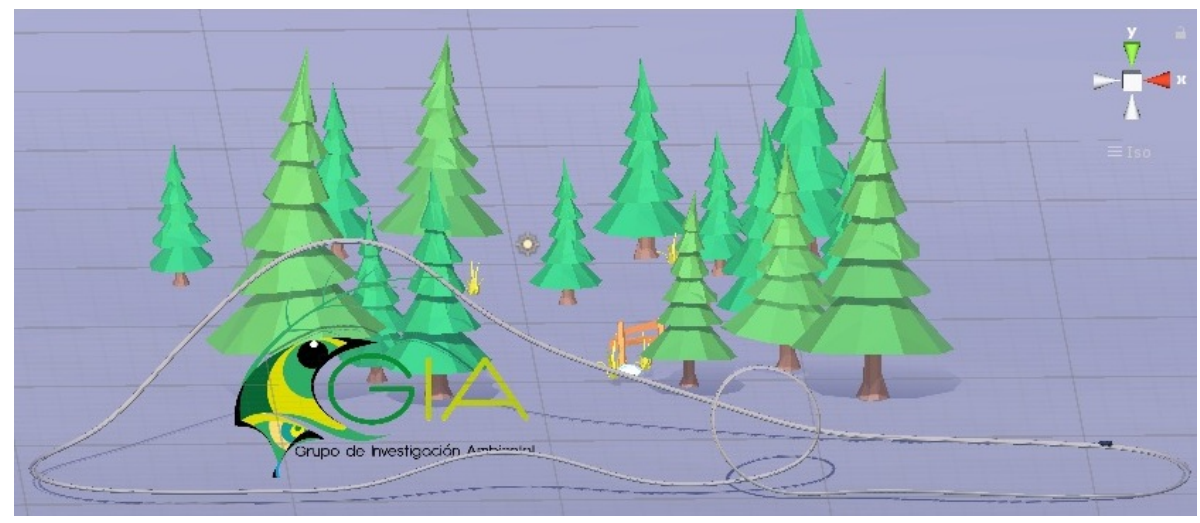

Figura 4: Vista panorámica del recorrido en montaña rusa bajo RV con textura.

observar todo el entorno que lo rodea, en frente de él se le presentan algunos marcadores y tableros en los que se evidencia el cambio de velocidad y altura con respecto a la base de la montaña. Desde el punto de vista más simple se le presentan cálculos de las variaciones de energía potencial y cinética del carrito asumiendo algunos valores estándar de la masa que este podría llegar a tener, así mismo, no se considera la fricción entre los rieles y el carrito. Los marcadores tienen disposición para enfrentar al estudiante a preguntas en relación a la energía cinética y potencial en determinados puntos del recorrido de la montaña donde la acción de su cabeza acciona las respuestas indicadas en el tablero a fin de permitir la continuación del recorrido o entregar un GAME OVER (Figura 5).

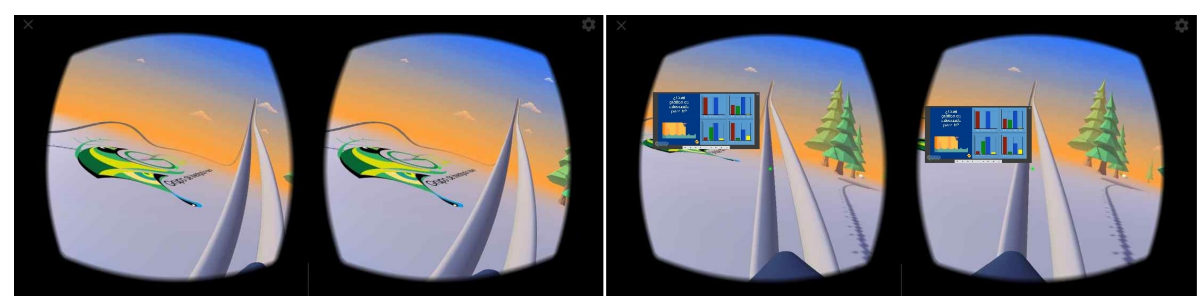

Figura 5: Vista estereoscópica del usuario. A la izquierda visión del usuario sobre el carrito en la montaña rusa; A la derecha interacción con preguntas de tipo gráficos en barras.

- Concepto de energía en el entorno de la RA

Para la RA tomando en consideración que esta permite una interacción con el entorno se crea algo parecido a una clase guiada, donde se le presenta al usuario un recorrido histórico del concepto, la interacción con objetos en caída libre (Figura 7 y 8), un abordaje del concepto visto desde punto de vista de las interacción de la materia con su entorno, la visualización grafica de las variaciones de la energía mecánica, un test de abordaje previo y otro de profundización. También es de destacar que dentro de este entorno todo lo interactivo se manipula a partir de punteros similares al mouse de una computadora.

3.2.4. Prueba de la herramienta didáctica basada en $R V$ y $R A$ Durante el desarrollo del proyecto se realizó una serie de pruebas en cuanto a la programación y configuración de cada uno de los parámetros (gráficos, interactivos, conceptuales), una vez se determinó los parámetros para su 


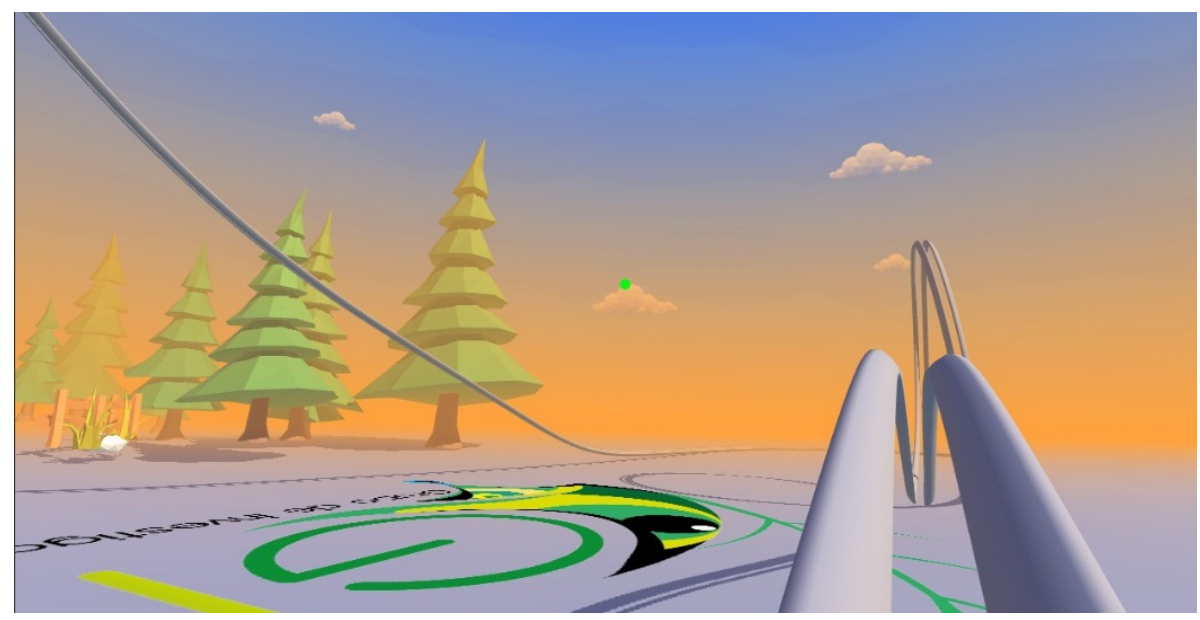

Figura 6: Vista panorámica del usuario sobre el carrito.

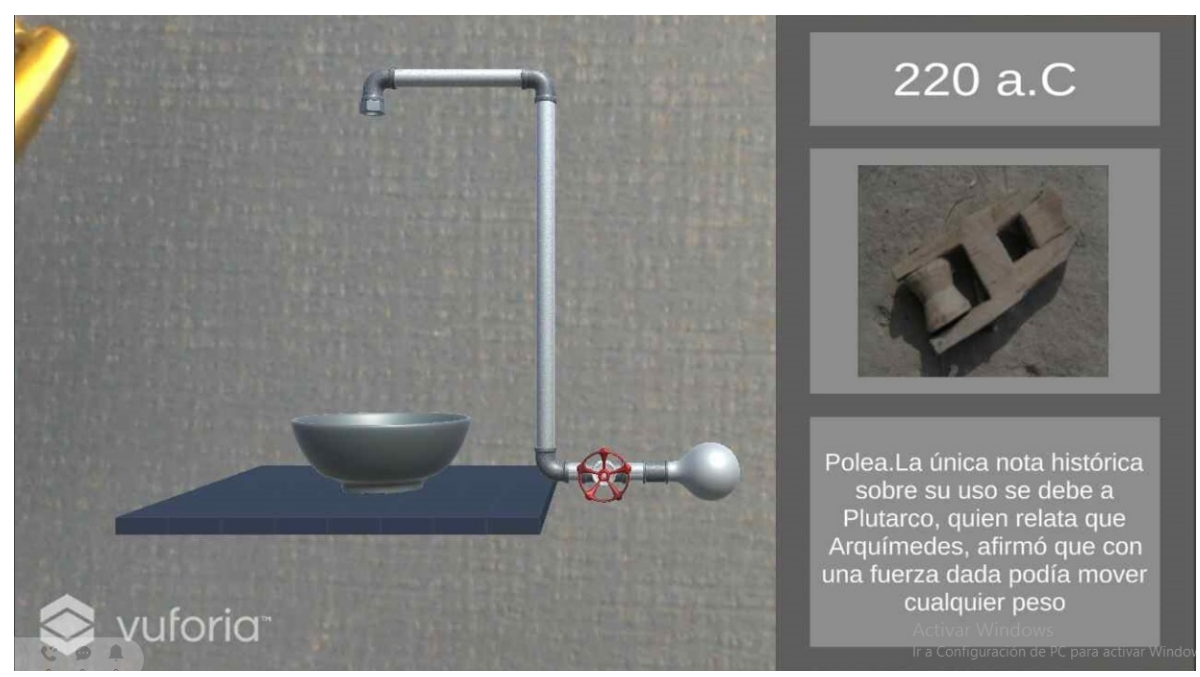

Figura 7: A la izquierda, recipiente que recibe objetos en caída libre; a la derecha, dato histórico de la polea.

programación y al completar la configuración, se desarrolló la primera aplicación de prueba con realidad aumentada denominada "Prototipo1".

Para llegar a este resultado se tuvo en consideración diversos ensayos que permitieron identificar la manera adecuada como ajustar la herramienta didáctica con funciones básicas y un contenido esencial para la apropiación del conocimiento en cada estudiante.

\subsection{Etapa 3. Evaluación de los resultados parciales en una prueba piloto}

Así como lo mencionan Contreras, Eguia y Solano, "la participación significa que en el proceso están involucrados no sólo los investigadores profesionales, sino la comunidad destinataria del proyecto, que no son considerados como simples objetos de investigación sino como sujetos activos que contribuyen a transformar la realidad", con ello se hicieron pruebas con usuarios y se evaluaron criterios iguales a las mencionan estos autores en su trabajo, estas pruebas permiten evaluar criterios encaminados a la inmersión, la satisfacción, aprendizaje, efectividad, motivación, emoción y socialización [30]. Se utilizó un cuestionario a manera de encuesta como herramienta de evaluación, con el objetivo de conocer la opinión y la efectividad de la 


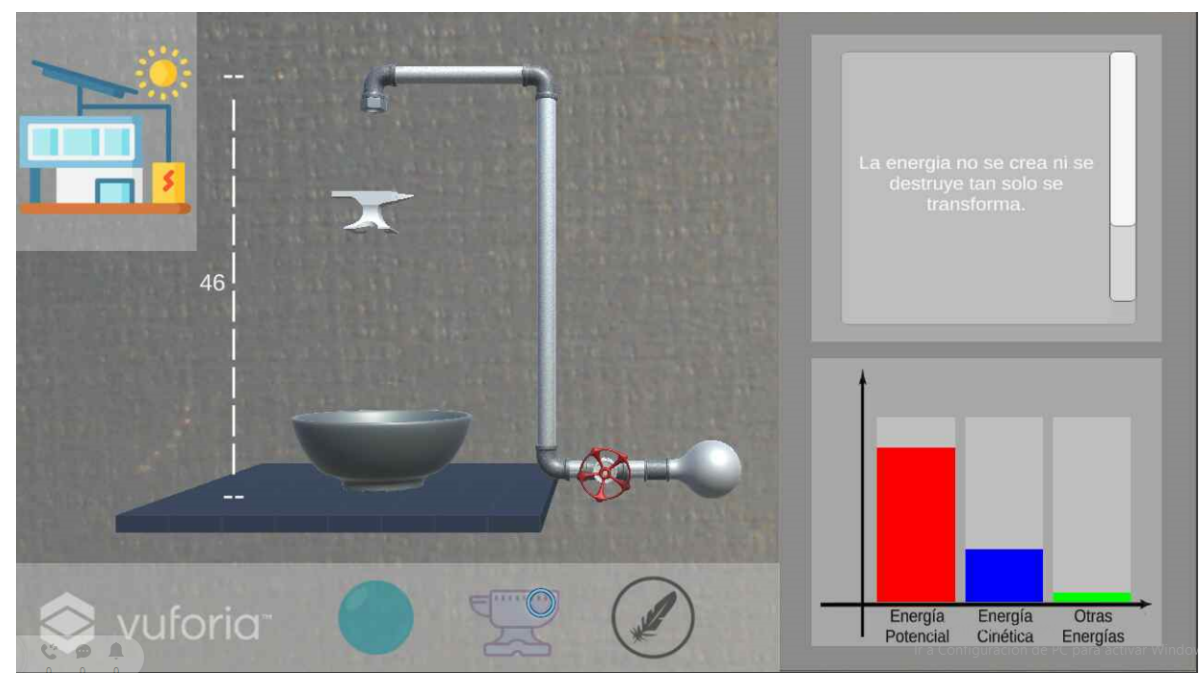

Figura 8: A la izquierda, interacción con tres objetos (pelota, yunque y pluma) en caída libre; a la derecha, dato teórico en relación a energía y debajo gráficas en barras de la variación de energía de los objetos en caída libre.

herramienta. Este cuestionario se aplicó a pocos docentes y estudiantes después de utilizar la herramienta, y contenían preguntas cerradas y abiertas.

Para evaluar la potencialidad de la unidad didáctica basada en RV y RA se toma en cuenta las opiniones que mostraron los docentes y estudiantes que hicieron parte de la prueba piloto en relación a los criterios antes mencionados, demostrando que tiene una aceptación significativa sobre los estudiantes, declarando lo bueno en su uso y aplicación. Este resultado es comparable con investigaciones como la de González y Chávez [27], donde señalan que "La herramienta muestra su potencialidad como tecnología de nueva generación, ya que se adapta a los canales de comunicación del alumno, lo que facilita el nivel de retención de la información, que mejora la comunicación en el proceso de enseñanza-aprendizaje".

Por parte de los docentes se enfatiza la posibilidad de ser adaptada a sus propios cursos dentro de los conceptos más complejos que no se pueden visualizar dentro de la química o la biología. Conociendo que los estudiantes poseen diversas habilidades de aprendizaje y que muchas de ellas pueden ser estimuladas por medio de dispositivos, la sensibilidad de la tecnología RV y RA muestra ventajas en donde es destacable el uso multisensorial, siendo este uno de los puntos fuertes de esta herramienta enfatizada en el aprovechamiento en el grado de retención de la información. Adicional a esto, el poder recolectar la información que los estudiantes entregan a través de la vinculación de los dispositivos a internet, permite al docente un diagnóstico rápido de la apreciación conceptual sobre el concepto trabajado.

Para desarrollar de manera más eficiente la etapa final del proyecto, se aplicaran encuestas sobre un número significativo de usuarios (estudiantes) que hagan uso de esta tecnología y que a partir de las respuestas que puedan recolectar, se logre medir el nivel de satisfacción, claridad, retroalimentación, los niveles de dificultad, la sensibilidad y la apropiación conceptual.

\section{Conclusiones}

A partir de esta investigación se demuestra que si se puede realizar la búsqueda de una necesidad dentro del aula de clase y que esto permite observar todas las posibilidades de solución. Se puede concluir que hoy en día se es necesario una articulación entre el lenguaje con el que se comunican los estudiantes y el que domina los textos educativos. La aparición de las TIC y con ellas las tecnologías de RV y RA, muestran que son un espacio en el que se puede interactuar 
de manera amigable y se les puede entregar información digerible a los estudiantes.

En el mercado existen un número considerable de opciones que permiten la interacción con la RV y RA, así mismo con esta investigación se encontró que al programar se puede abordar de múltiples maneras un determinado concepto o fenómeno, todo queda en la disposición de tiempo y alcances económicos.

Es necesario explorar el contenido conceptual que se desea impartir dentro de estas plataformas, de modo que se pueda ajustar un contenido digerible para el usuario sin llegar a agobiar y fatigar con el uso prolongado de estos dispositivos.

La exploraron de cómo se construyen unidades didácticas es un fuerte de esta investigación, ya que articula el contenido de manera secuencial como si se estuviese en el aula de clase, lo que permite una entrega de contenidos y actividades en concordancia con lo que se desea enseñar. En este sentido también es importante explorar que es lo sabe el estudiante y como este método aporta a mejorar y acercar al estudiante a un concepto más científico.

\section{Referencias}

[1] I. San Mauro M., M. González F., and L. Collado Y., "Aplicaciones móviles en nutrición, dietética y hábitos saludables; análisis y consecuencia de una tendencia a la alza," Nutr. Hosp., vol. 30, pp. 15-24, 2014

[2] H. Moreno-Casado, R. Cuevas, J. J. Pulido Gonzáles, and T. García Cavlo, "Influencia de una aplicación de telefonía móvil de entrenamiento sobre las necesidades psicológicas y la motivación autodeterminada en escolares," Cuad. Psicol. Del Deport., vol. 15, pp. 71-78, 2015.

[3] L. E. Galván Rico and R. E. Reyes Gil, "Algunas herramientas para la prevención, control y mitigación de la Contaminación ambiental," Universidad, Cienc. Y Tecnol., pp. 287-294, 2009.

[4] H.-M. Marroquín-Yeroví, "Docentes estratégicos forman estudiantes estratégicos: Una propuesta pedagógica para el trabajo de aula," Editor. Publicaciones UNIMAR-, 2013.

[5] J. Moreno and V. Valderrama, "Aprendizaje Basado en Juegos Digitales en Niños con TDAH: un Estudio de Caso en la Enseñanza de Estadística para Estudiantes de Cuarto Grado en Colombia," Rev. Bras. Educ. Espec., pp. 143-158, 2015.

[6] V. Potkonjak et al., "Virtual laboratories for education in science, technology, and engineering: A review.," Comput. Educ., vol. 95, pp. 309-327, 2016.

[7] M. Picquart, “¿Qué podemos hacer para lograr un aprendizaje significativo de la física?," Lat. Am. J. Phys. Educ., vol. 2, no. 1, pp. 29-36, 2008.

[8] O. E. Tamayo, "Representaciones semióticas y evolución conceptual en la enseñanza de las ciencias y las matemáticas," Rev. Educ. y Pedagog., vol. XVIII, no. 45, pp. 39-49, 2006.

[9] J. de A. Pacca and K. F. Henrique, "Dificultades y estrategias para la enseñanza del concepto de energía," Enseñanza las ciencias Rev. Investig. y Exp. didácticas, vol. 22, no. 1, pp. 159-166, 2004.

[10] J. Doménech et al., "La enseñanza de la energíA: Una propuesta de debate para un replanteamiento global," Cad. Bras. Ensino Física, vol. 20, pp. 285-311, 2003.

[11] E. Alomá and M. Malaver, "Aaálisis de los conceptos de energía, calor, en textos universitarios de termodinámica," Enseñanza de las ciencias, vol. 25(3), pp. 387-400, 2007.

[12] B. Follari, M. T. Perrotta, G. N. Dima, and E. E. Gutiérrez, "Una aplicación del teorema de conservacion de la energía como problema integrador," Rev. Bras. Ensino Física, vol. 33, no. 1, pp. 1-6, 2011.

[13] M. T. Perrotta et al., "La Energía . Planificación , aplicación y evaluación de una Estrategia Didáctica para un curso universitario de Física Básica en carreras de Ciencias Naturales," Latin-American J. Phys. Educ., vol. 3, 2009.

[14] H. D. Young and R. A. Freedman, Física Universitaria Vol.1, 12th ed., vol. 1 y 2. México: Pearson Educación, 2009.

[15] J. J. G. García and F. J. P. Perales, “¿Cómo usan los profesores de Química las representaciones semióticas?," Rev. Electrónica Enseñanza las Ciencias, vol. 5, no. 2, pp. 247-259, 2006.

[16] A. Hernandez, J. Cervantes, J. Ordoñez, and M. del S. Gracia, "Teoría de registros de representaciones semiótica," no. April, 2017.

[17] L. M. Oviedo, A. M. Kanashiro, M. Bnzaquen, and M. Gorrochategui, "Los registros semióticos de representación en matemática," Aula Univ., no. 13, pp. 29-36, 2014.

[18] C. A. Solano Villanueva, J. F. Casas Díaz, and J. C. Guevara Bolaños, "Aplicación móvil de realidad aumentada para la enseñanza de la clasificación de los seres vivos a niños de tercer grado.," 2015.

[19] T. de Monterrey, "Realidad aumentada y virtual," 2017. [Online]. Available: https://goo.gl/X3F3UK. 
[20] P. O. Valencia-Vallejo, N. G., Huertas-Bustos, A. P., Baracaldo-Ramírez, "Los ambientes virtuales de aprendizaje: una revisión de publicaciones entre 2003 y 2013, desde la perspectiva de la pedagogía basada en la evidencia," Rev. Colomb. Educ., 2014.

[21] D. J. Restrepo Durán, L. S. Cuello Montañez, and L. D. C. Contreras Chinchilla, "Juegos didácticos basados en realidad aumentada como apoyo en la enseñanza de biología," Ingeniare, no. 19, p. 99, 2015.

[22] C. E. O. Rangel, "Realidad aumentada en medicina," Rev. Colomb. Cardiol., vol. 18, no. 1, pp. 4-7, 2011.

[23] E. Martelo, M. Manotas, and B. Vallejo, "Prototipo De Una Aplicación Móvil Con Realidad Aumentada Para Mostrar Puntos De Información De Ubicación De La Universidad Simón Bolívar En Barranquilla Colombia Mediante El Uso Del Navegador Móvil Junaio," Investig. e Innovación en Ing., vol. 2, no. 2, pp. 29-34, 2014.

[24] L. Navarrete N, S. L. Alvarez P, M. Folrez P, V. Camelo A, and E. Gonzalez Q, "Evaluación de la integración de realidad aumentada en un curso básico de laboratorio de mecánica," Innovación docente y uso las TIC en Educ., pp. 1-13, 2017.

[25] O. E. Tamayo, M. Orrego, and F. J. Ruiz, Unidades didacticas para la enseñanza de las ciencias. Manizales, 2016.

[26] J. Ramos, "Realidad aumentada como estrategia didáctica, para la enseñanza y aprendizaje en el área de ética y valores con los estudiantes del grado sexto, en el colegio nacional universitario de Vélez.," 2017.

[27] A. González and G. Chávez, "La Realidad Virtual Inmersiva En Ambientes Inteligentes De Aprendizaje," vol. 2, pp. 122-137, 2011.

[28] M. N. Cordoba y C. Monsalve, «TIPOS DE INVESTIGACIÓN: Predictiva, proyectiva, interactiva, confirmatoria y evaluativa».

[29] Muy interesante, «Muy Interesante,» [En línea]. Available: https://www.muyinteresante.es/tecnologia/articulo/realidadaumentada-la-ultima-revolucion-digital. [último acceso: 7 2020].

[30] R. S. Contreras Espinosa, J. L. Eguia Gómez y L. Solano Albajes, «Videojuegos como un entorno de apendizaje,» ICONO 14, vol. 2, nº 9, pp. 249-261, 2011.

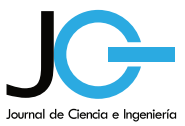

